\title{
Transferrin receptor targeting nanomedicine delivering wild-type p53 gene sensitizes pancreatic cancer to gemcitabine therapy
}

\author{
ER Camp ${ }^{1,2}$, C Wang $^{1}$, EC Little ${ }^{3}$, PM Watson ${ }^{4}$, KF Pirollo $^{5}$, A Rait ${ }^{5}$, DJ Cole ${ }^{1}$, EH Chang ${ }^{5}$, \\ and DK Watson ${ }^{4,6}$ \\ ${ }^{1}$ Department of Surgery, Medical University of South Carolina, Charleston, SC, USA \\ ${ }^{2}$ Ralph H Johnson VA Medical Center, Chärleston, SC, USA \\ ${ }^{3}$ Department of Microbiology and Immunology, Medical University of South Carolina, Charleston, \\ SC, USA \\ ${ }^{4}$ Department of Pathology and Laboratory Medicine, Medical University of South Carolina, \\ Charoleston, SC, USA \\ ${ }^{5}$ Department of Oncology, Lombardi Comprehensive Cancer Center, Georgetown University \\ Medical Center, Washington, DC, USA \\ ${ }^{6}$ Department of Biochemistry and Molecular Biology, Medical University of South Carolina, \\ Medical University of South Carolina, Charleston, SC, USA
}

\begin{abstract}
To overcome gene therapy barriers such as low transfection efficiency and nonspecific delivery, liposomal nanoparticles targeted by a single-chain antibody fragment to the transferrin receptor (TfRscFv) delivering wild-type (wt) human p53 (SGT-53) were developed for tumor-specific targeting, We hypothesize that SGT-53 in combination with gemcitabine will demonstrate enhanced therapeutic benefit in an in vivo metastatic pancreatic cancer model. Intrasplenic injection of $1 \times 10^{6}$ Panc 02 murine pancreatic cancer cells was used to generate in vivo hepatic metastatic tumors. Nanoparticle localization was assessed by tail vein injection of TfRscFv with fluorescently labeled oligonucleotides (6-carboxyfluorescein phosphoramidite (6FAM) ODN) imaged by Xenogen IVIS 200 scan. SGT-53 (equivalent to $30 \mu \mathrm{g}$ of $p 53$ intravenously) and gemcitabine $(20 \mathrm{mg} / \mathrm{kg}$ Intraperitoneally) alone and in combination were administered biweekly and compared with untreated mice. Survival was determined by blinded daily assessment of morbidity. Human wt $p 53$ expression and transferrin levels in the tumors were assessed by western blot analysis. Tumor burden was quantified by liver weight, Xenogen imaging demonstrated tumor-specific uptake of TfRscFv-6FAM ODN. Exogenous human wt $p 53$ protein was detected in the SGT-53-treated tumors compared with control. Compared with untreated mice with metastatic tumors demonstrating median survival of 20 days, SGT-53, gemcitabine and the combination demonstrated improved median survival of 29,30 and 37 days, respectively. The combination treatment prolonged median survival when compared with single drug treatment and decreased tumor burden. The tumor targeting liposomal-based SGT-53 nanoparticle is capable of sensitizing pancreatic cancer to conventional chemotherapy in pancreatic cancer models. This approach has
\end{abstract}

(C) 2013 Nature America, Inc. All rights reserved

Correspondence: ER Camp, Associate Professor Department of Surgery, Medical University of South Carolina, 25 Courtenay Drive, Room 7018, MSC795, Charleston, SC 29425, USA. campe@ musc.edu.

CONFLICT OF INTEREST Drs Chang and Pirollo are two of the inventors of the described technology, for whom several patents owned by Georgetown University have been issued. The patents have been licensed to SynerGene Therapeutics for commercial development. Dr Chang owns equity interests in SynerGene and serves as a non-paid scientific consultant to SynerGene. 
the potential to be translated into a new, more effective therapy for pancreatic cancer. Further optimization is ongoing, moving towards a Phase $1 \mathrm{~B} / 2$ clinical trial.

\section{Keywords}

pancreatic cancer; transferrin; p53; nanoparticle

\section{INTRODUCTION}

With nearly 44000 estimated new cases with 37000 estimated deaths in 2012, pancreatic adenocarcinoma remains the fourth leading cause of cancer-related deaths in the United States, highlighting the devastating nature of this malignancy. ${ }^{1}$ Over the past few decades, mortality from breast, colon, lung and prostate cancer has steadily decreased. Conversely, mortality from pancreas cancer has remained relatively stable since the 1950s owing to the lack of significant progress in the fight against pancreatic cancer. ${ }^{1}$

Even with optimal surgery and aggressive adjuvant therapy, overall survival from pancreatic cancer remains poor with reported 5-year survival rates of only $15-20 \% .^{2}$ Although surgery is the only curative option, $<20 \%$ of patients are eligible for surgery owing to metastatic spread at the time of diagnosis. ${ }^{3}$ Pancreatic cancer patients with metastatic spread at diagnosis have a dismal survival of $<9$ months. ${ }^{4}$ Gemcitabine chemotherapy remains a primary option for patients with metastatic pancreatic cancer. ${ }^{3,5}$ However, gemcitabine only prolongs survival approximately 6 months, nearly 2 months longer than 5-fluorouracil in multiple Phase III clinical trials and only extended 1 year survival to $18 \% .^{6,7}$ The exceedingly poor prognosis for patients with pancreatic cancer necessitates further development of novel therapeutic approaches incorporating gene therapy strategies.

Critical hurdles still exist preventing effective gene therapy in vivo and, more importantly, in the clinic. These include low transfection efficiency, poor tissue penetrance as well as nonspecific delivery. ${ }^{8}$ To overcome these barriers, many strategies have been developed, such as viral, liposome and polyethyleneimine delivery mechanisms. However, these strategies are primarily non-targeted, resulting in poor efficiency and significant nonspecific gene silencing. ${ }^{8}$ Viral delivery strategies have improved transfection efficiency, although they lack specific targeting and the viral particles can have residual immunologic effects. Cationic liposomes composed of positively charged lipid bilayers can be used to delivery gene therapy, but similarly lack specificity.

Specific targeted vector strategies are appealing to improve delivery directly and more efficiently to the tumor. The transferrin (Tf) receptor has been investigated as a potential target for vector delivery in gene therapy with growing support. ${ }^{9}$ In pancreatic cancer, the Tf receptor was overexpressed in $93 \%$ of the pancreatic tumor cells relative to normal tissue, suggesting that it may be a specific marker for malignancy. ${ }^{10} \mathrm{~A}$ second factor supporting the Tf receptor as an appropriate target in pancreatic cancer is that the Tf receptor is recycled during internalization in rapidly dividing cancer cells, thus improving uptake of Tf-targeted vectors. ${ }^{8}$

Recently, a nanoparticle liposome-based complex targeting the Tf receptor has been used to target specifically tumors for gene therapy. ${ }^{8,11-15}$ In this complex, the payload is encapsulated within a cationic liposome, the surface of which is decorated with an anti-Tf receptor single-chain antibody fragment (TfRscFv) targeting moiety. When systemically administered, this self-assembled, biodegradable, nanosized complex has been shown to preferentially target and efficiently deliver gene therapies, not only to primary tumors but 
also to metastatic lesions (including in the brain) in animal models, delivering plasmid DNA carrying normal human $p 53$ gene, ${ }^{14,15}$ antisense HER-2, ${ }^{16}$ chemotherapeutic agents, ${ }^{17}$ small interfering RNAs, ${ }^{11}$ and magnetic resonance imaging contrast agents, gadolinium ${ }^{12,18}$ and superparamagnetic iron oxide. ${ }^{19,20}$ Although taken up by cells of the reticuloendothelial system of the liver and lung, as it is not a sterically stabilized particle, this nanocomplex does not transfect the hepatocytes or lung aveolar cells themselves. $8,11,12,19$

P53 restoration has been shown most effective in enhancing cytotoxicity when used in combination with an agent that results in DNA damage or initiates apoptosis ${ }^{21,22}$ Given the proapoptotic and antineoplastic effects of $p 53$ restoration therapy, we hypothesized that SGT-53 treatment should increase the responsiveness of pancreatic cancer tumor cells to gemcitabine and, therefore, enhance gemcitabine therapy in a metastatic pancreatic cancer model.

\section{MATERIALS AND METHODS}

\section{Cell lines and culture conditions}

Murine Panc02 cells were obtained from the NCI DCTD Tumor Repository (NCI, Frederick, MD, USA). Panc02 murine pancreatic cancer cells were maintained in RPMI 1640 supplemented with $10 \%$ fetal bovine serum. Human pancreatic adenocarcinoma AsPC-1, BxPC-3, Capan-2, HPAC, MIA PaCa-2 and PANC-1 were maintained in RPMI 1640 (AsPC-1, BxPC-3 and Capan-2) or Dulbecco's modified Eagle's medium (HPAC, MIA $\mathrm{PaCa}-2$ and PANC-1) supplemented with $10 \%$ fetal bovine serum. Mycoplasma-negative cultures were ensured by polymerase chain reaction testing using MycoSensor PCR assay kit (Agilent Technologies, La Jolla, CA, USA) before the investigations. Cells were monitored throughout the course of these studies and demonstrated consistent morphology and doubling time.

\section{Reagents}

TfRscFv-Lip carrying a 6-carboxyfluorescein phosphoramidite (6FAM)-labeled phosphorothioate, non-sequence-specific, scrambled ( $5^{\prime}$-6FAM-

CTAGCCATGCTTGTC-3') oligonucleotides (6FAM-ODN) (TriLink Biotechnologies, San Diego, CA, USA) and TfRscFv-Lip-p53 (SGT-53) were prepared as described previously. ${ }^{15,16,23}$ Briefly, the cationic lipid consisted of a 1:1 molar ratio of dioleoyltrimethylammonium propane and the fusogenic neutral lipid dioleolyphosphatidylethanolamine prepared by the ethanol injection method. ${ }^{24}$ Cytomegalovirus promoter controlled plasmid containing the wild-type (wt) $p 53$ was used to deliver the gene therapy. The complex is prepared by simple mixing of the components at a defined ratio in a specific order. ${ }^{23}$ Previously, the SGT-53 particle was optimized with DNA/lipid ratio of 1:14 $\mu \mathrm{g} \mathrm{nmol}^{-1}$ and TfRscFv protein/lipid ratio of $20 \mu \mathrm{g} \mu \mathrm{mol}^{-1}$ The SGT-53 immunolipoplex particle has a size $<400 \mathrm{~nm}$ (number average) as measured on a Malvern Nano ZS (Malvern Instruments, Malvern, UK) with a net positive charge between 20 and $40 \mathrm{mV} .{ }^{19}$ The immunolipoplex preparations were lyophilized and stored at $-20^{\circ} \mathrm{C}$. For injection, the samples were reconstituted by the addition of the appropriate volume of phosphate-buffered saline (PBS).

Goat anti-rabbit and horse anti-mouse horseradish peroxidase-conjugated antibodies were acquired from Cell Signaling Technology (Danvers, MA, USA). Mouse monoclonal antibody against $\mathrm{Tf}$ receptor was obtained from Life Technologies (Grand Island, NY, USA). Mouse monoclonal antibody against p53 (DO-7) was obtained from Santa Cruz Biotechnologies (Santa CruZ, CA, USA). Rabbit polyclonal antibody against $\beta$-actin was obtained from Sigma (St Louis, MO, USA). 


\section{Western blot analysis}

Cultured cells were suspended in radioimmunoprecipitation assay protein lysis buffer $(\mathrm{pH}$ 7.4), containing $20 \mathrm{~m}_{\mathrm{M}}$ sodium phosphate, $150 \mathrm{~m}_{\mathrm{м}}$ sodium chloride, $1 \%$ Triton $\mathrm{X}-100,5 \mathrm{mм}$ ethylenediaminetetraacetic acid, 5mм phenylmethylsulfonyl fluoride, $1 \%$ aprotinin, $1 \mathrm{mg} \mathrm{ml}$ leupeptin and $500 \mathrm{~m}_{\mathrm{M}} \mathrm{Na}_{3} \mathrm{VO}_{4}$. Tumor sections were dissected and homogenized in ice-cold radioimmunoprecipitation assay buffer ( $3 \mathrm{ml}$ radioimmunoprecipitation assay buffer per $\mathrm{g}$ wet tissue). After $30 \mathrm{~min}$ incubation on ice, tissue debris was removed by centrifugation at $10000 \mathrm{~g}$ for $10 \mathrm{~min}$ at $4{ }^{\circ} \mathrm{C}$. Protein concentration was quantified using Pierce BCA protein assay kit (Thermo Scientific, Rockford, IL, USA). In all, $20 \mu \mathrm{g}$ of total protein from cultured cells or $200 \mu \mathrm{g}$ of tissue extracts was resolved with sodium dodecyl sulfate polyacrylamide gel electrophoresis (10\% polyacrylamide gel), and transferred to Amersham Hybon-ECL membrane (GE Healthcare, Piscataway, NI USA). Blots were probed with mouse monoclonal antibodies against $p 53$ (1:200 dilution) or Tf receptor (1:500 dilution) in $5 \%$ non-fat milk at $4{ }^{\circ} \mathrm{C}$ for $16 \mathrm{~h}$. All membranes were stripped and reprobed with $\beta$-actin antibody for loading control. Membranes were developed using the SuperSignal West Pico Chemiluminescent Substrate (Thermo Scientific).

\section{Flow cytometry}

Single-cell suspensions of murine Panc02 cells were stained with phycoerythrin-conjugated anti-CD71 (Tf receptor) antibody. Live-gated cells were analyzed with the FlowJo software (Treestar, Ashland, OR, USA) for CD71 expression compared with unstained controls.

\section{Cell growth assay}

For the in vitro proliferation assays, Panc02 cells were seeded in flat-bottom, 96-well plates (2000 cells per well) in RPMI 1640 plus 10\% fetal bovine serum. After $24 \mathrm{~h}$, the cells were treated with $50 \mu \mathrm{l}$ of serum-free medium containing SGT-53 (equivalent to $100 \mathrm{ng} p 53$ ). After $4 \mathrm{~h}$ incubation, $100 \mu \mathrm{l}$ of RPMI 1640 plus $10 \%$ fetal bovine serum containing $0,0.2$ and $0.5 \mu_{\mathrm{M}}$ gemcitabine was added to each well. At $72 \mathrm{~h}$ post-treatment, $10 \mu \mathrm{l}$ of a 1-(4,5dimethythiazol-2-yl)-3,5-diphenyltetrazolium bromide dye solution (5 mg ml; Sigma, St Louis, MO, USA) was added to each well, and the plate was incubated at $37^{\circ} \mathrm{C}$ for $4 \mathrm{~h}$. Reactions were stopped by the addition of $100 \mu \mathrm{l}$ of a $10 \%$ sodium dodecyl sulfate $/ 0.01 \mathrm{~m}$ $\mathrm{HCl}$ solution. After an additional overnight incubation, absorbance at $570-630 \mathrm{~nm}$ was recorded on a BioTek microtiter plate reader (Winooski, VT, USA).

\section{In vivo metastatic pancreatic cancer model}

C57BL/6 mice (8-12 weeks old) were used for these experiments, which were performed according to protocols approved by the Institutional Animal Care and Use Committee at the Medical University of South Carolina. This model has been previously described in detail. ${ }^{25}$ Briefly, following anesthesia, $1 \times 10^{6}$ Panc02 cells in $0.1 \mathrm{ml}$ PBS (Hyclone, Logan, UT) were injected using a $28-\mathrm{G}$ needle and syringe directly into the tip of the exteriorized spleen through a $1 \mathrm{~cm}$ left subcostal incision. Animals were monitored daily for health, and moribundity was determined by a blinded investigator. Untreated mice become moribund at an average of 18-20 days after tumor injection. In all cases, animals were killed if evidence of pain or suffering was present.

\section{Assessment of nanoparticle tumor targeting}

TfRscFv-Lip-6FAM-ODN particle was assessed for tumor localization based on Xenogen imaging. Mice with advanced tumors were either tail vein injected with TfRscFvLip-6FAM-ODN or PBS (Hyclone) on day 14 after intrasplenic cancer cell injection with imaging performed $12 \mathrm{~h}$ later. Fluorescence of the liver and spleen was captured on CCD camera (excitation 445-490 nm/emission 515-575 nm) and analyzed. 


\section{Treatment schedule}

For therapeutic assessment of TfRscFv-Lip p53 (SGT-53) in combination with gemcitabine, we used a $20 \mathrm{mg} / \mathrm{kg}$ intraperitoneally gemcitabine dose delivered two time weekly. ${ }^{25}$ SGT-53 (370 $\mu \mathrm{l}$ suspended in PBS, equivalent to $30 \mu \mathrm{g}$ of $p 53)$ was administered via tail vein injection two times weekly. This treatment schedule was based on the previously determined timing of the expression of the exogenous $p 53$ in mouse tumor models. ${ }^{26}$ Gemcitabine administration was initiated at $48 \mathrm{~h}$ after SGT-53 treatment. Both SGT-53 and gemcitabine treatments continued for 4 weeks.

\section{In vivo therapeutic end points}

Two independent in vivo studies using the metastatic pancreatic cancer model were performed to assess the efficacy of the Tf receptor-targeted $p 53$ gene therapy combined with gemcitabine. In the first experiment, SGT-53 treatment was initiated on day 5 after tumor implantation. In total, 7 mice were used in each treatment group except the combination group with 10 mice. Mice were treated for 4 weeks and then observed. Mice were closely followed and humanely killed when morbidity was determined by an investigator in a blinded manner. In a parallel tumor quantitative experiment, SGT-53 treatment was initiated on day 8 after tumor implantation. We terminated the experiment on day 19 after killing all the experimental mice at the same time point for molecular and quantitative tumor analysis.

\section{Immunohistochemistry}

Tumors were fixed in 10\% buffered formalin, set in paraffin blocks and processed into 5$\mu \mathrm{m}$-thick sections. Sections were stained with standard hematoxylin and eosin. Terminal deoxynucleotidyi transferase dUTP nick-end labeling (TUNEL) was performed according to the manufacturer's directions (DeadEnd Colorimetric TUNEL System; Promega, Madison, WI, USA). TUNEL-positive cells were quantified at $\times 400$ magnification, with three random fields counted per section. Antigen retrieval was performed before Ki-67 staining by immersing the slides in $10 \mathrm{~m}_{\mathrm{M}}$ citrate buffer ( $\mathrm{pH}$ 6.0, Antigen Unmasking Solution; Vector Labs, Burlingame, CA, USA) and microwaving for $10 \mathrm{~min}$. Sections were stained with antiKi-67 antibody (Abeam, Cambridge, MA; no. 15580) overnight at 1:750 dilution. Secondary antibody and DAB chromogen detection was performed following the manufacturer's protocol (Rabbit IgG Vectastain Elite ABC kit; Vector Labs). Ki-67-positive cells were counted at $\times 400$ magnification, with at least three random fields counted per section.

\section{Statistical analysis}

Statistical analysis was performed using the GraphPad Prism 5 software (La Jolla, CA, USA). Kaplan-Meier survival analysis was used for the metastatic morbidity study. Linear regression analysis was used to identify correlations between bioluminescence total flux and tumor weight, with a $P$-value $<0.05$ considered statistically significant for identifying the existence of a relationship and a goodness-of-fit $R^{2}$ value to determine the extent of said relationship. Two-tailed unpaired Student's $t$-tests were used for statistical analysis in all other experiments, with a $P$-value $<0.05$ considered statistically significant.

\section{RESULTS}

Expression of Tf receptor in a panel of pancreatic cancer cell lines $\mathrm{Tf}$ receptor was expressed in all pancreatic cancer cell lines tested (Figure 1). Variable expression was observed with the HPAC, MIA PaCa-2 and PANC-1 cells demonstrating the highest expression relative to the other cell lines. The murine Panc02 cells also demonstrated high Tf receptor expression compared with the human pancreatic cancer cell lines. Cell surface 
Tf receptor expression was confirmed in Panc02 cells using flow cytometric analysis compared with unstained controls.

\section{In vitro efficacy of SGT-53 in combination with gemcitabine}

Compared with untreated control group, SGT-53 treatment alone had no effect on the growth of Panc 02 cells. Gemcitabine treatment $\left(0.5 \mu_{\mathrm{M}}\right)$ demonstrated $19 \%$ decrease in growth. The combination of SGT-53 and $0.5 \mu_{\mathrm{m}}$ gemcitabine inhibited cell growth by $49 \%$ (Figure 2; $P<0.001$ vs control, SGT-53 alone and $0.5 \mu_{\mathrm{M}}$ gemcitabine alone), Gemcitabine $\left(0.2 \mu_{\mathrm{M}}\right)$ did not significantly decrease cellular growth. However, the addition of SGT-53 with $0.2 \mu_{\mathrm{M}}$ gemcitabine inhibited growth by $35 \%$ (Figure 2; $P<0.01$ vs control, SGT-53 alone and $0.5 \mu_{\mathrm{M}}$ gemcitabine alone). Prior investigations have established the cancer cell targeting ability and specificity of this TfRscFv complex. Previously performed in vitro competition studies with the addition of free TfRscFv effectively inhibited the transfection activity of TfRscFv complexed nanoparticles. The observed effect was dose-dependent, suggesting that the demonstrated transfection efficiency is mediated through targeting the $\mathrm{Tf}$ receptor. ${ }^{14,15,27}$

\section{Tumor localizing ability of the TfRscFv-LipA nanoparticle to hepatic metastatic pancreatic cancer tumors}

In our metastatic pancreatic cancer model, we assessed tumor targeting of the TfRscFvLip-6FAM ODN, Tumor targeting was assessed at a late tumor growth time point (14 days after splenic injection) to determine tumor penetration at an advanced tumor stage. On the basis of ex vivo organ Xenogen IVIS 200 imaging $12 \mathrm{~h}$ after tail vein injection of TfRscFvLip-6FAM ODN particle compared with saline control, a significant increase in signaling intensity was observed both in the tumor-bearing liver and splenic tissue (Figure 3a). Fluorescence intensity analysis demonstrated a $>3$-fold increase intensity in the tum or bearing tissues injected with TfRscFv-Lip-6FAM ODN compared with control (Figures $3 b$ and c). This result demonstrates the tumor-specific targeting ability of the TfRscFv-Lip nanoparticle in our metastatic pancreatic cancer model. Previously performed in vivo experiments also incorporated a control single-chain antibody fragment (which does not target the $\mathrm{Tf}$ receptor), as the targeting moiety demonstrating no tumor-targeting specificity compared with the TfRscFv complexed nanoparticles, suggesting that the tumor-targeting ability is specific to the TfR antibody fragment. ${ }^{14}$

\section{In vivo efficacy of SGT-53 in combination with gemcitabine in a metastatic pancreatic cancer model}

We assessed the efficacy in two separate in vivo experiments using the described metastatic pancreatic cancer model, TfRscFv-LipA without $p 53$ has previously shown no efficacy relative to untreated control. ${ }^{28}$ Therefore, for these experiments, PBS was used as a control group. In the first experiment, we assessed long-term survival based on mouse morbidity. Treatment was continued for a total of 4 weeks and then the mice were observed for signs of morbidity. In a second experiment using the same in vivo model, all treatments groups were killed at the same time point 19 days following tumor implantation. This was performed to compare treatment group in regards to $p 53$ expression and to assess the effect of treatment on tumor burden (liver weight).

In the survival experiment, SGT-53 combined with gemcitabine significantly extended the time to observed morbidity. Untreated mice demonstrated signs of morbidity starting on day 20 after tumor implantation with a median survival time of 21 days. Compared with untreated control group, both gemcitabine and SGT-53 as single agents extended survival with a median survival of 30 and 29 days, respectively (Figure $4 ; P<0.01$ vs control). The combination of gemcitabine and SGT-53 extended survival compared with untreated and 
individual treatment arms with a median survival of 37 days (Figure 4; $P<0.01$ vs all comparison groups). Of note, in the combination arm, no mouse showed signs of morbidity while on treatment. However, after 4 weeks, once treatment was discontinued, the mice rapidly demonstrated significant morbidity related to tumor progression, suggesting that the treatment effectively suppressed growth in this aggressive metastatic pancreatic cancer model.

In the second experiment, all groups were terminated on day 19 after tumor implantation when control mice demonstrated signs of increasing morbidity to allow for comparison of tumor burden and for tumor molecular analysis. To demonstrate SGT-53 delivery, we assessed exogenous human-specific $p 53$ protein expression by western blot analysis. Compared with untreated and gemcitabine-treated tumors, SGT-53-treated tumors demonstrated exogenous human $p 53$ expression (Figure $5 \mathrm{a}$ ). The presence of exogenous human $p 53$ demonstrates successful delivery of the SGT-53 complex. Owing to the efficacy of the combination treatment in which no significant tumor burden was evident on day 19 , this group could not be assessed for the presence of exogenous $p 53$.

Liver weight in grams $(\mathrm{g})$ was significantly increased in the untreated tumor-bearing mice $(2.68 \pm 0.27 \mathrm{~g})$ compared with normal mouse liver (without tumor; $1.16 \pm 0.08 \mathrm{~g})(P<0.05)$. Average liver weight in the SGT-53 $(2.22 \pm 0.52 \mathrm{~g})$, gemcitabine alone $(2.14 \pm 0.28 \mathrm{~g})$ and in the combination treatment group (SGT-53/gemcitabine; $1.61 \pm 0.12 \mathrm{~g}$ ) were decreased compared with untreated control. (Figure 5b). The combination group demonstrated the lowest tumor burden compared with the other groups based on liver weight $(P<0,05)$.

TUNEL staining demonstrated significantly increased tumor cell apoptosis in the combination treatment group. No significant differences were observed in the untreated, gemcitabine and SGT-53 treatment groups, with an average of 5,5 and 8 apoptotic cells per high-power field identified, respectively (Figures $5 \mathrm{c}$ and d). However, in the combination group, 22 apoptotic cells per high-power field were identified, suggesting an increase in apoptosis compared with the other groups possibly explaining the observed differences in liver tumor burden $(P<0.001)$, No differences were noted in proliferation based on Ki-67 staining (data not shown).

\section{DISCUSSION}

The exceedingly poor prognosis for patients with pancreatic cancer necessitates further development of novel therapeutic approaches, including novel gene therapy strategies. To overcome barriers preventing effective gene therapy, a liposome-based nanocomplex targeting the $\mathrm{Tf}$ receptor using an anti-Tf receptor single-chain antibody fragment (TfRscFvLip) have been used to target tumors specifically. $8,13,14,26,28,29$ This promising strategy has demonstrated effectiveness delivering $p 53$ therapy (SGT-53) in various preclinical cancer models. ${ }^{14,26,28,29}$ Previously published results using this nanodelivery system have demonstrated its in vivo targeting specificity in delivering 6FAM-ODN to breast cancer metastatic lesions in the lungs in which only the tumor nodule displayed a high level of fluorescence with no significant signal in the adjacent normal lung tissue. ${ }^{11}$ Similarly, in an investigation using an orthotopic prostate cancer model, systemically delivered TfRscFvLip-6FAM-ODN was visualized by fluorescence signal only in the primary tumor without uptake in the normal liver. ${ }^{8}$ In vivo studies incorporating a control single-chain antibody fragment failed to demonstrate tumor targeting compared with the TfRscFv-Lip nanoparticle. These previous in vivo experiments along with previously performed in vitro competition studies using free $\mathrm{TfRscFv}$ demonstrate compelling evidence that the targeting ability of the TfRscFv-Lip nanoparticle is specifically mediated through the Tf receptor. 
The current investigation demonstrates that the nanocomplex-based p53 gene therapy can enhance gemcitabine efficacy in an immunocompetent metastatic pancreatic cancer model. The combined therapy extended mouse survival, decreased tumor burden and increased tumor cell apoptosis. Furthermore, we demonstrated enhanced tumor expression of human p53 protein by western blot analysis confirming effective gene therapy delivery. Tumor localization of the TfRscFv-LipA particles was demonstrated based on Xenogen imaging of the 6FAM-ODN particle.

Our results highlight the clinical potential of molecular-based tumor targeting nanoparticles as gene therapy vehicles as well as the therapeutic benefit of $p 53$ gene therapy to enhance current chemotherapy efficacy. Given the effectiveness of $p 53$ restoration in enhancing cytotoxicity when used in combination with an agent, which results in DNA damage or initiates apoptosis, we hypothesized that SGT-53 would increase the efficacy of gemcitabine. ${ }^{21,22}$ Although the combination of gemcitabine with SGT-53 extended survival significantly compared with untreated and individual treatment groups, the difference was much less than that observed in prior investigations using SGT-53. The aggressive tumor model used in the current investigation may explain the observed decreased efficacy. This metastatic pancreatic cancer model reflects the devastating nature of pancreatic cancer with mice becoming moribund on average of 19 days after tumor injection demonstrating tumor burden completely replacing the liver. ${ }^{25}$ This development of tumor burden reflects the rapid decline in health we observe in the mice as tumors progress. Although the combined treatment only modestly prolonged survival compared with individual treatment arms, survival time was almost doubled relative to the control group. It should be noted that the treatment in the studies described here was only for 4 weeks. Based on the observation that minimal or no tumor burden was evident in the mice treated with the combination with SGT-53 and gemcitabine by day 19, continued treatment has the potential to increase survival even further.

This investigation highlights the potential of systemically delivered gene therapy using a Tf receptor-targeted nanoparticle to enhance current therapeutic strategies in pancreatic cancer. The ability to deliver effectively tumor-targeted nanoparticles as demonstrated in our investigations may open the door for novel nanoparticle-based therapeutic applications in pancreatic cancer. Furthermore, a Phase I clinical trial in patients with advanced solid tumors was recently completed with a single agent SGT-53. The single agent was well tolerated, with the majority of patients demonstrating stable disease following treatment. The promising Phase I results combined with our preclinical data support the development of Phase Ib/II clinical trials in pancreatic cancer evaluating the combination therapy.

\section{CONCLUSION}

Wtp53 gene therapy via the systemically administered Tf receptor-targeted nanocomplex can effectively target pancreatic cancer tumors and enhance gemcitabine chemotherapy. Our findings support the development of pancreatic cancer Phase Ib/II clinical trials using this combined therapeutic strategy as a new potentially more effective treatment for pancreatic cancer.

\section{Acknowledgments}

This work was supported in part by the Flow Cytometry and Cell Sorting and Molecular imaging Shared Resource, Hollings Cancer Center, Medical University of South Carolina (P30 CA138313) and National Institutes of Health, 5R01CA123159-05 (DJC, DKW) and 1K08CA142904 (ERC). 


\section{REFERENCES}

1. Siegel R, Naishadham D, Jemal A. Cancer statistics, 2012. Cancer J Clinl. 2012; 62:10-29.

2. Winter JM, Cameron JL, Campbell KA, Arnold MA, Chang DC, Coleman J, et al. 1423 pancreaticoduodenectomies for pancreatic cancer: a single-institution experience. J Gastrointest Surg. 2006; 10:1199-1210. discussion 1210-1. [PubMed: 17114007]

3. American Cancer Society. Cancer Facts \& Figures. American Cancer Society; Atlanta, GA, USA: 2010.

4. Saif MW. Pancreatic neoplasm in 2011: an update. J Pancreas. 2011; 12:316-321.

5. Moss RA, Lee C. Current and emerging therapies for the treatment of pancreatic cancer. Onco Targets Ther. 2010; 3:111-127. [PubMed: 20856847]

6. Burris HA 3rd, Moore MJ, Andersen J, Green MR, Rothenberg ML, Modiano MR, et al. Improvements in survival and clinical benefit with gemcitabine as first-line therapy for patients with advanced pancreas cancer: a randomized trial. J Clin Oncol. 1997; 15:2403-2413. [PubMed: 9196156]

7. Ishii H, Furuse J, Nagase M, Yoshino M. Impact of gemcitabine on the treatment of metastatic pancreatic cancer. J Gastroenterol Hepatol. 2005; 20:62-66. [PubMed: 15610448]

8. Pirollo KF, Zon G, Rait A, Zhou Q, Yu W, Hogrefe R, et al. Tumor-targeting nanoimmunoliposome complex for short interfering RNA delivery. Hum Gene Ther. 2006; 17:117-124. [PubMed: 16409130]

9. Gatter KC, Brown G, Trowbridge IS, Woolston RE, Mason DY. Transferrin receptors in human tissues: their distribution and possible clinical relevance. J Clin Pathol. 1983; 36:539-545.

[PubMed: 6302135]

10. Ryschich E, Huszty G, Knaebel HP, Hartel M, Buchler MW, Schmidt J. Transferrin receptor is a marker of malignant phenotype in human pancreatic cancer and in neuroendocrine carcinoma of the pancreas. Eur J Cancer. 2004; 40:1418-1422. [PubMed: 15177502]

11. Pirollo KF, Rait A, Zhog Q, Hwang SH, Dagata JA, Zon G, et al. Materializing the potential of small interfering RNA via a tumor-targeting nanodelivery system. Cancer Res. 2007; 67:29382943. [PubMed: 17409398]

12. Pirollo KF, Dagata J, Wang P, Freedman M, Vladar A, Fricke S, et al. A tumor-targeted nanodelivery system to improve early MRI detection of cancer. Mol Imag. 2006; 5:41-52.

13. Pirollo KF, Rait A, Zhou Q, Zhang XQ, Zhou J, Kim CS, et al. Tumor-targeting nanocomplex delivery of novel tumor suppressor RB94 chemosen sitizes bladder carcinoma cells in vitro and in vivo. Clin Cancer Res. 2008; 14:2190-2198. [PubMed: 18381961]

14. Xu L, Huang CC, Huang W, Tang WH, Rait A, Yin YZ, et al. Systemic tumor-targeted gene delivery by anti-transferrin receptor scFv-immunoliposomes. Mol Cancer Ther. 2002; 1:337-346. [PubMed: 12489850]

15. Xu L, Tang WH, Huang CC, Alexander W, Xiang LM, Pirollo KF, et al. Systemic p53 gene therapy of cancer with immunollpoplexes targeted by anti-transferrin receptor scFv. Mol Med. 2001; 7:723-734. [PubMed: 11713371]

16. Rait AS, Pirollo KF, Xiang L, Ulick D, Chang EH. Tumor-targeting, systemically delivered antisense HER-2 chemosensitizes human breast cancer xenografts irrespective of HER-2 levels. Mol Med. 2002; 8:475-486. [PubMed: 12435858]

17. Hwang SH, Rait A, Pirollo KF, Zhou Q, Yenugonda VM, Chinigo GM, et al. Tumor-targeting nanodelivery enhances the anticancer activity of a novel qutnazolinone analogue. Mol Cancer Therap. 2008; 7:559-568. [PubMed: 18347143]

18. Freedman M, Chang EH, Zhou Q, Pirollo KF. Nanodelivery of MRI contrast agent enhances sensitivity of detection of lung cancer metastases. Acad Radiol. 2009; 16:627-637. [PubMed: 19345904]

19. Yang C, Rait A, Pirollo KF, Dagata JA, Farkas N, Chang EH. Nanoimmunoiiposome delivery of superparamagnetic iron oxide markedly enhances targeting and uptake in human cancer cells in vitro and in viva. Nanomed Nanotechnol Biol Med. 2008; 4:318-329. 
20. Dagata JA, Farkas N, Dennis CL, Shull RD, Hackley VA, Yang C, et al. Physical characterization methods for iron oxide contrast agents encapsulated within a targeted liposome-based delivery system. Nanotechnology. 2008; 19:305101. [PubMed: 21828753]

21. Riva CM. Restoration of wild-type p53 activity enhances the sensitivity of pleural metastasis to cisplatin through an apoptotic mechanism. Anticancer Res. 2000; 20:4463-4471. [PubMed: 11205289]

22. Martins CP, Brown-Swigart L, Evan Gl. Modeling the therapeutic efficacy of p53 restoration in tumors. Cell. 2006; 127:1323-1334. [PubMed: 17182091]

23. Yu W, Pirollo KF, Yu B, Rait A, Xiang L, Huang W, et al. Enhanced transfection efficiency of a systemically delivered tumor-targeting immunolipoplex by inclusion of a $\mathrm{pH}$-sensitive histidylated oligolysine peptide. Nucleic Acids Res. 2004; 32:e48. [PubMed: 15026537]

24. Campbell MJ. Lipofection reagents prepared by a simple ethanol injection technique. BioTechniques. 1995; 18:1027-1032. [PubMed: 7546703]

25. Little EC, Wang C, Watson PM, Watson DK, Cole DJ, Camp ER. Novel immuno-competent murine models representing advanced local and metastatic pancreatic cancer. J Surg Res. 2012; 176:359-366. [PubMed: 22221605]

26. Xu L, Pirollo KF, Chang EH. Transferrin-liposome-mediated p53 sensitization of squamous cell carcinoma of the head and neck to radiation in vitro. Hum Gene Ther. 1997; 8:467-475. [PubMed: 9054521]

27. Yu W, Pirollo KF, Rait A, Yu B, Xiang LM, Huang WQ, et al. A stericalty stabilized immunolipoplex for systemic administration of a therapeutic gene. Gene Therapy. 2004; 11:14341440. [PubMed: 15229629]

28. Xu L, Pirollo KF, Chang EH. Tumor-targeted p53-gene therapy enhances the efficacy of conventional chemo/radiotherapy. J Control Rel. 2001; 74:115-128.

29. Xu L, Pirollo KF, Tang WH, Rait A, Chang EH. Transferrin-liposome-mediated systemic p53 gene therapy in combination with radiation results in regression of human head and neck cancer xenografts. Hum Gene Ther. 1999; 10:2941-2952. [PubMed: 10609655] 

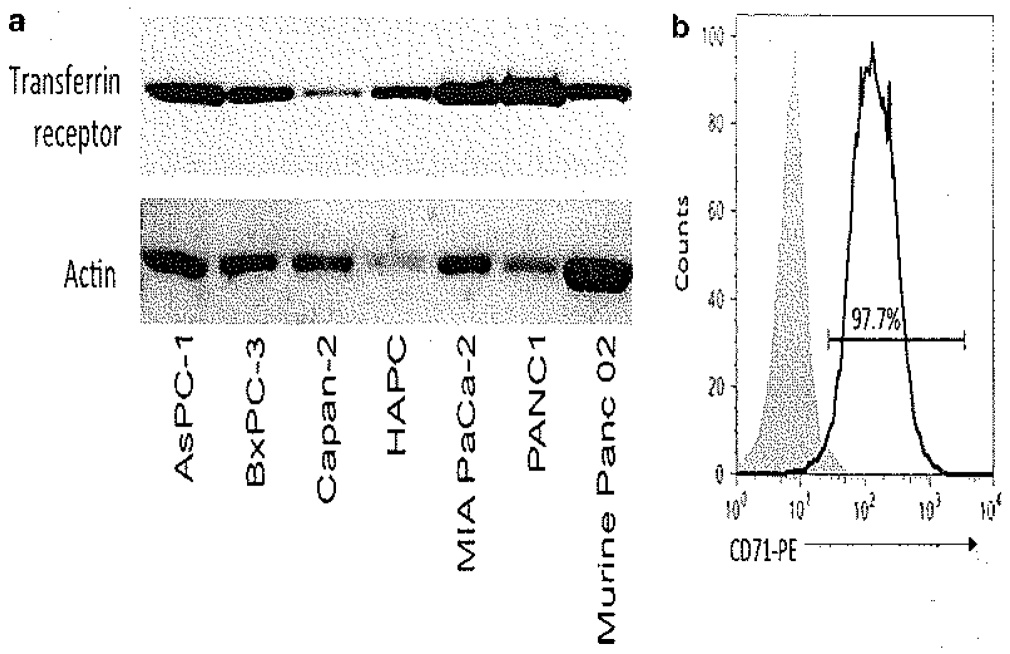

Figure 1.

Pancreatic cancer cell lines express the transferrin receptor, (a) Western blot analysis demonstrated consistent expression of transferrin receptor across a panel of pancreatic cancer cell lines, including the murine Panc02 pancreatic cancer cells. Actin was used as a loading control, (b) Single-cell suspensions of murine Panc02 cells were stained with phycoerythrin (PE)-conjugated anti-CD71 (transferrin receptor) antibody and compared with unstained control cells for flow analysis. 


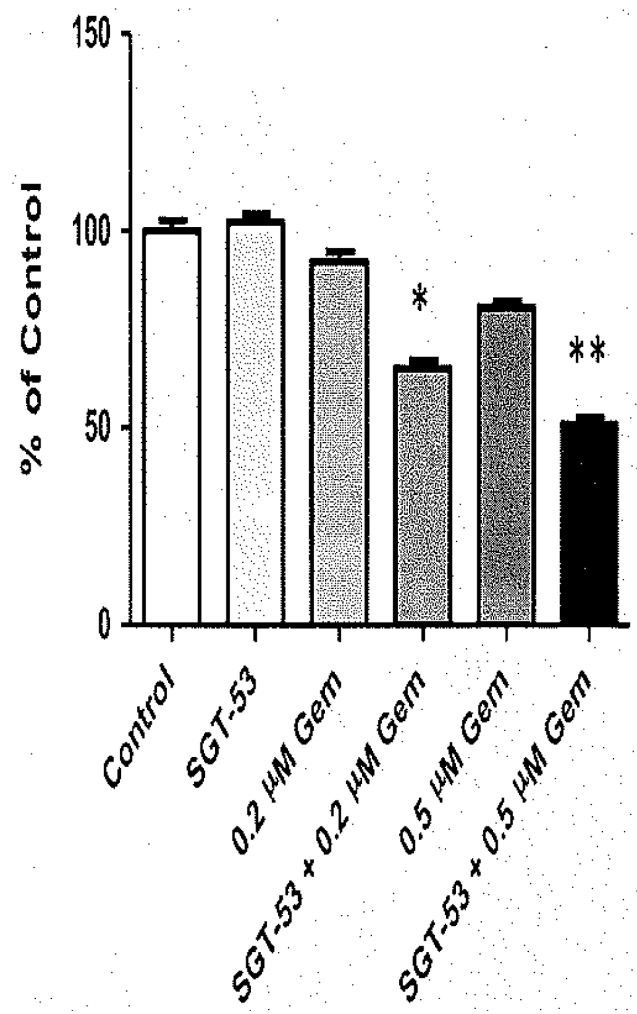

Figure 2.

SGT-53 enhanced gemcitabine (Gem) in vitro growth inhibition. At $72 \mathrm{~h}$ following treatment, Panc02 cells demonstrated significantly decreased cell growth with the combination of Gem and SGT-p53. * $P<0.01 ; * * P<0.001$. 


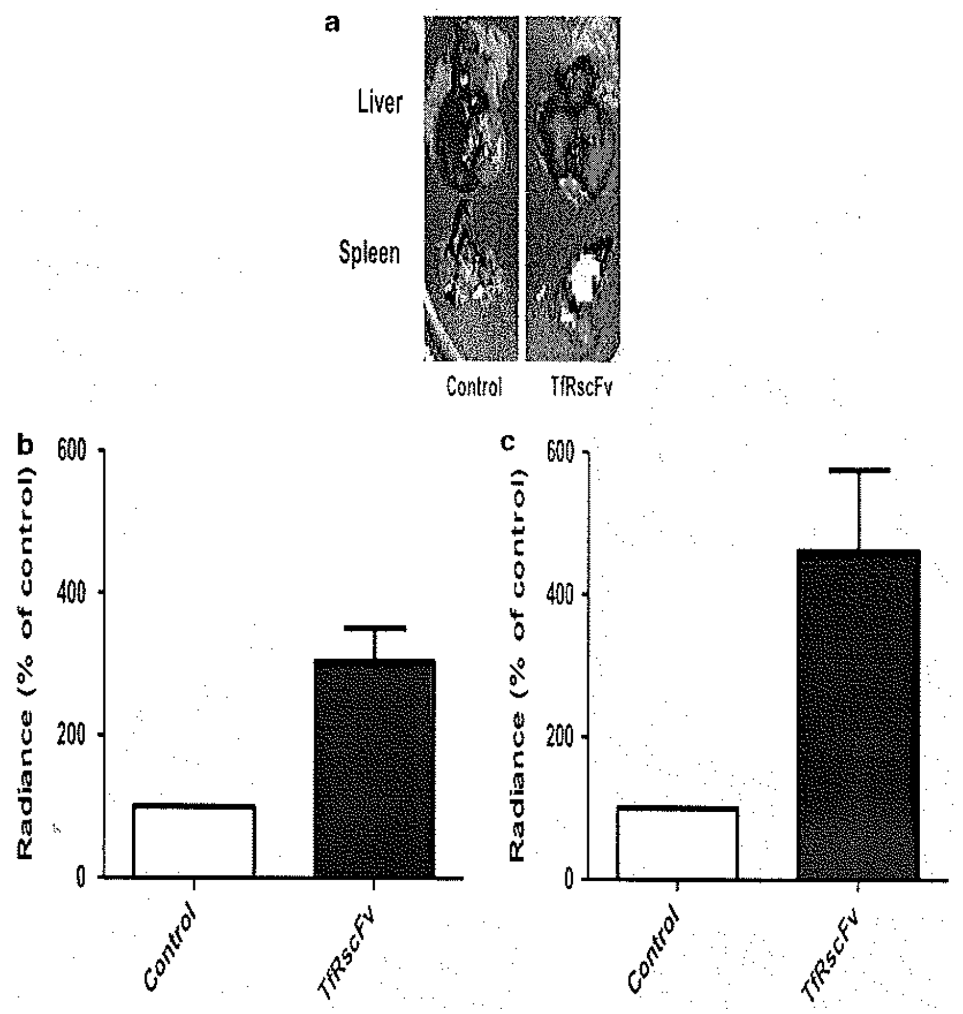

Figure 3.

Tf receptor single-chain antibody fragment (TfRscFv)-Lip nanoparticles demonstrate tumor targeting in the metastatic pancreatic cancer in vivo model. At day 14 after intrasplenic Panc02 cell injection, TfRscFv-Lip-6FAM oligonucleotide (ODN) nanoparticles were delivered systemically by tail vein injection. Groups receiving TfRscFv-Lip-6FAM ODN were compared with saline control, (a) Representative Xenogen images obtained $12 \mathrm{~h}$ after injection demonstrated significant increase in fluorescent intensity in the TfRscFv-

Lip-6FAM ODN mouse group. Fluorescence intensity analysis demonstrated a $>3$-fold increased intensity in the tumor-bearing liver (b) and spleen (c) from mice intravenously injected with TfRscFv-Lip-6FAM ODN compared with control. Lip, liposome. 


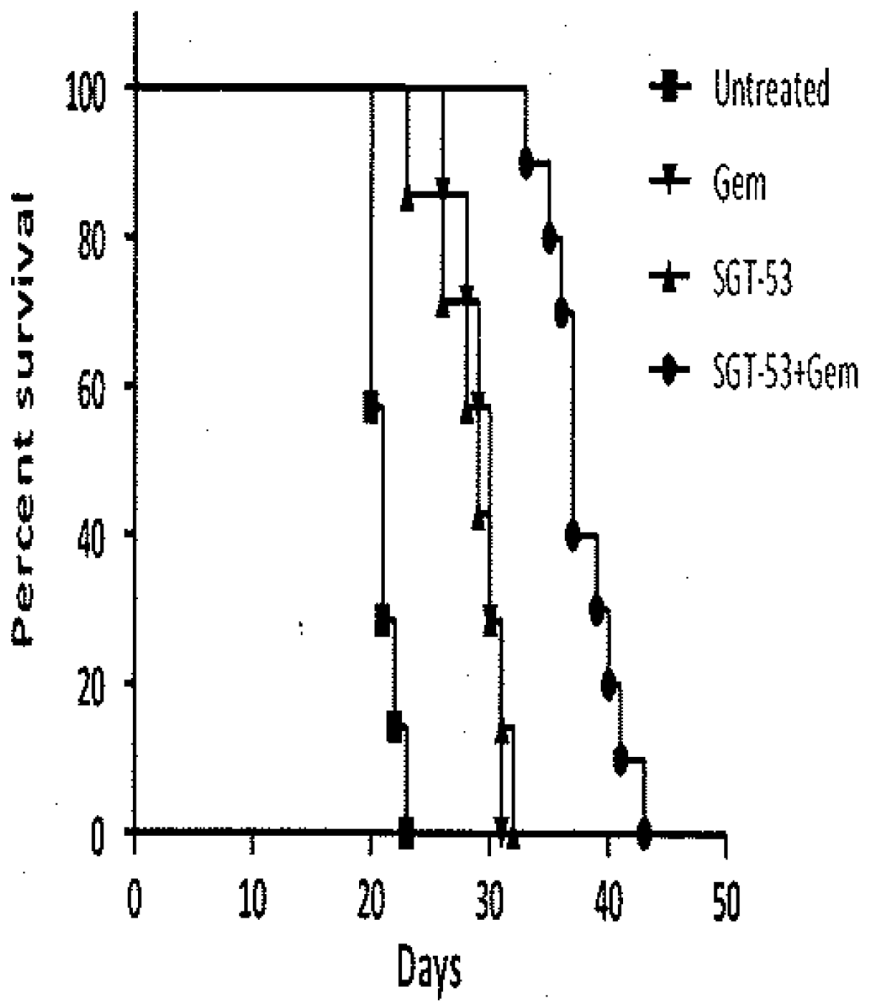

Figure 4.

Combination gemcitabine (Gem) and SGT-53 significantly enhanced survival in a metastatic pancreatic cancer model. Survival based on mouse morbidity was significantly extended in the combination group by 7-8 days compared with individual treatment groups and by 16 days compared with untreated controls $(P<0.01)$. The figure demonstrates Kaplan-Meier survival curves for treatment groups. 
a Humanp 53 Actin
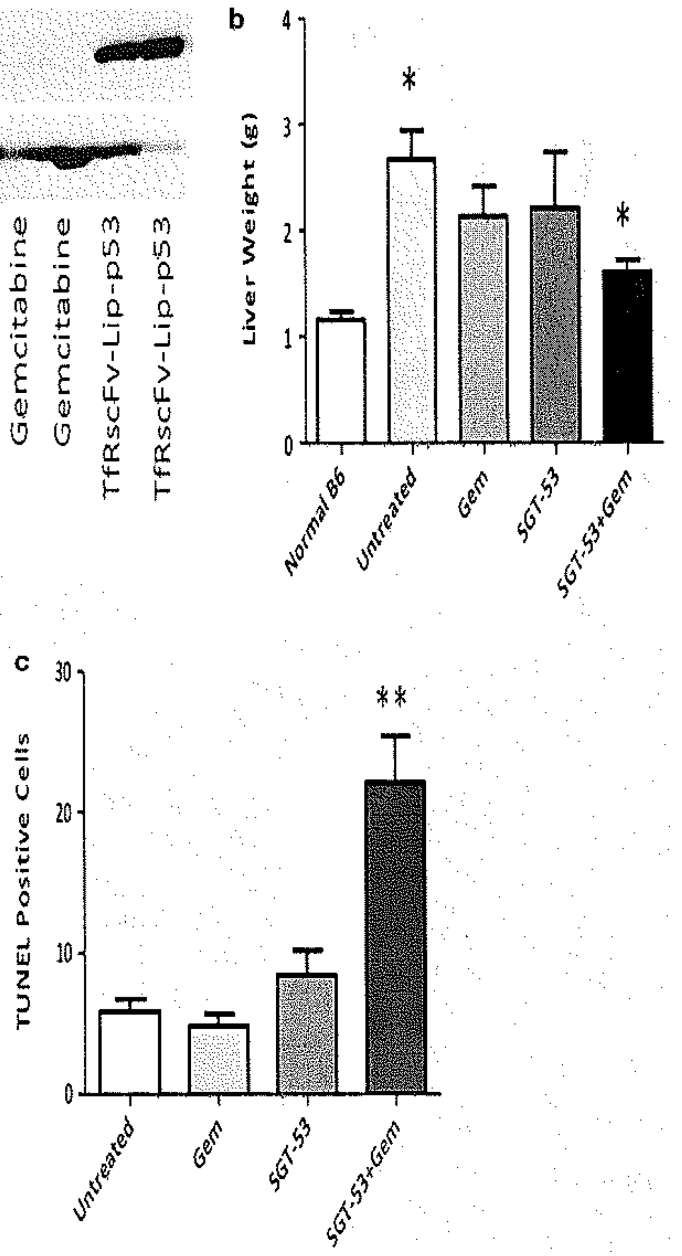

Figure 5.

Combination gemcitabine (Gem) and SGT-53 significantly decreased murine metastatic pancreatic cancer tumor burden and enhanced tumor apoptosis, Treatment groups were evaluated at day 19 after intrasplenic Panc02 cell injection, (a) Human p53 protein expression was observed only in the SGT-53-treated group by western blot, (b) Significant differences in liver weight were demonstrated in the combination group associated with (c) enhanced apoptosis based on the terminal deoxynucleotidyl transferase dUTP nick-end labeling (TUNEL) assay. $* P<0.05 ; * * P<0.001$. 